\title{
Evaluación por y desde los usuarios: objetos de aprendizaje con Realidad aumentada
}

\section{Evaluation by and from users: learning objects with Augmented Reality}

\author{
Julio Cabero-Almenara \\ Universidad de Sevilla. Sevilla, España \\ cabero@us.es \\ Carmen Llorente-Cejudo \\ Universidad de Sevilla. Sevilla, España \\ karen@us.es \\ Juan Jesús Gutiérrez-Castillo \\ Universidad de Sevilla. Sevilla, España \\ jjesusgc@us.es
}

\begin{abstract}
Resumen
Bajo el objetivo de conocer las valoraciones que los estudiantes, que habían utilizado objetos de aprendizaje en realidad aumentada, realizaban de los mismos, se diseñó un estudio "expost-facto" en el que participaron 429 estudiantes, los cuales cursaban las asignaturas de "Tecnología Educativa" y "TIC aplicadas a la educación" del Grado de Educación Infantil y Educación Primaria de la Facultad de Ciencias de la Educación, de la Universidad de Sevilla.

Para conocer las valoraciones de los sujetos, se diseñó un cuestionario "ad hoc" con construcción tipo Likert que perseguía recoger información sobre tres dimensiones: aspectos técnicos y estéticos del objeto producido en RA, facilidad de utilización, y guía elaborada para facilitar la comprensión del funcionamiento del objeto por los estudiantes. Las valoraciones positivas realizadas por parte de los estudiantes, entre otras, nos permiten señalar que estos recursos pueden ser válidos para su incorporación en los procesos de enseñanza-aprendizaje.
\end{abstract}

\section{Palabras clave}

Realidad Aumentada, Diseño, producción y evaluación de medios y materiales, Experiencia en el aula, Integración curricular de las TIC, Tecnologías emergentes.

\footnotetext{
Abstract

With the objective of knowing the assessments that the students, who had used learning objects in augmented reality, made of them, an "ex-post-facto" study was designed in which 429 students participated in the subjects of "Educational Technology" and "ICT applied to Education" attended the Primary and Childhood Education of the Faculty of Education Sciences of the University of Seville.

In order to know the assessments of the subjects, an "ad hoc" questionnaire was designed with a Likert type construction that sought to gather information on three dimensions: technical and aesthetic aspects of the object produced in RA, ease of use, and a guide developed to facilitate the understanding of the Performance of the object by students.

The positive evaluations made by the students, among others, allow us to point out that these resources can be valid for their incorporation into the teaching-learning processes.

Key words

Augmented reality, Design, production and evaluation of means and materials, Experience in the classroom, Integration curricular of the ITC, Emergent technologies.
} 


\section{Introducción.}

Si bien es cierto que ya desde principios de la década de los 90 el término de Realidad Aumentada (RA) fue investigado y abordado a través de una gran variedad de campos, tales como la medicina, la aeronáutica, robótica o el turismo (Bower et al., 2014), también lo es que ha sido en los últimos años cuando ésta ha ido alcanzando un auge mayor, sobre todo en lo que a su incorporación a la formación. En este sentido, el sistema de RA ya establecía un proceso a través del cual combinar datos virtuales con datos del mundo real, lo que proporcionaba a los usuarios el acceso a un contenido multimedia rico, variado y significativo, facilitándole un contexto relevante y con el que poder interactuar de manera inmediata (Cabero \& García, 2016). Para ello, pueden incorporarse diferentes tipos de objetos como por ejemplo: imágenes fijas, videoclips, sonidos, texto, modelos $3 \mathrm{D}$, sitios web,...; todo ello para crear un nuevo escenario comunicativo.

\subsection{La Realidad Aumentada: tecnología emergente para la formación}

Así pues, la RA, por las numerosas posibilidades educativas que proporciona, ya se configuraba como un sistema con un inmenso potencial para mejorar el aprendizaje y la enseñanza, como así identificaban investigaciones realizadas al respecto (Billinghurst \& Duenser, 2012; Dede, 2009; Dunleavy, Dede, \& Mitchell, 2009; Johnson, Adams, \& Cummins, 2012; Kaufmann \& Schmalstieg, 2003; Shelton, 2002), y además, se presentan como apoyo y mejora de una variedad de enfoques pedagógicos, como por ejemplo:

a. Aprendizaje constructivista: mediante el uso de RA se motiva a los estudiantes a involucrarse de una manera más profunda con las tareas, conceptos y recursos estudiados a través del uso de superposiciones de información, permitiendo establecer conexiones más significativas y duraderas en lo que al conocimiento se refiere.

b. El aprendizaje contextual: el aprendizaje auténtico y contextualizado se posibilita mediante la incorporación de experiencias educativas en el entorno del mundo real, así como la introducción del mundo real en el aula.

c. Aprendizaje basado en juegos: los sistemas de realidad aumentada pueden usarse para facilitar el aprendizaje basado en juegos inmersivos creando una narrativa digital, colocando a los estudiantes en un rol, proveyendo recursos auténticos e incorporando información contextualmente relevante.

d. Aprendizaje basado en la investigación: ofreciendo un medio para recopilar electrónicamente datos para análisis futuros y proporcionar modelos virtuales situados dentro de un contexto real que son fácilmente manipulables, así como proporcionando información que se considera contextualmente relevante para el tema que se está investigando. (Johnson, Smith, Levine, \& Haywood, 2010).

Por otra parte, y como señalan diferentes autores (Santos, Chen, Taketomi, Yamamoto, Miyazaki, \& Kato, 2014; Bacca, Baldiris, Fabregat, Graf, \& Kinshuk, 2014; Barba, Yasaca, \& Manosalvas, 2015; Fonseca, Redondo \& Valls, 2015; Han, Jo, Hyun, \& So, 2015; Prendes, 2015; Reinoso, 2016; Santos et al., 2016), su incorporación a los

Evaluación por y desde los usuarios: objetos de aprendizaje con Realidad Aumentada. Julio Cabero Almenara, Carmen Llorente Cejudo, Juan Jesús Gutiérrez Castillo. 
procesos de enseñanza aprendizaje nos ofrece una serie de posibilidades, como son por ejemplo: enriquecer la información de la realidad para hacerla más comprensible al estudiante, facilitar el aprendizaje móvil y ubicuo, desarrollar escenarios formativos multimedia, eliminar de los escenarios formativos la información innecesaria que pueda impedir la observación de la información significativa, crear entornos activos de formación, aumenta la motivación del estudiante, observar los objetos desde múltiples perspectivas que son seleccionadas por los usuarios, y enriquecer los apuntes que les son facilitado a los estudiantes.

Desde una primera aproximación, y tal como apuntan Cabero y García (2016: 7), se puede decir que "se trata de una tecnología que permite la combinación de información digital e información física en tiempo real por medio de distintos soportes tecnológicos como por ejemplo las tablets o los smartphones, para crear con ello una nueva realidad enriquecida". Siendo también para estos autores sus características distintivas las de ser una realidad mixta, ofrecer en tiempo real la combinación de diferentes capas informativas, ser una tecnología interactiva, es fácil de manejar, y que mediante su utilización enriquecemos o alteramos la información de la realidad añadiéndole información adicional.

Su utilización se ve también ampliada, ya que pueden observarse en una diversidad de dispositivos, siendo los más usuales los smartphones, tecnología bastante común entre los alumnos. Ello repercute al mismo tiempo para que se potencie con ella el aprendizaje móvil y ubicuo (Vázquez-Cano \& Sevillano, 2015).

Cabría entonces hacernos una pregunta: ¿qué posibilidades incorpora la RA a la formación? Y al respecto y sintetizando las propuestas de diferentes autores algunas de ellas serían las siguientes: enriquecer la información de la realidad para facilitar su comprensión para el estudiante, potenciar la creación de entornos multimedia, facilitar el aprendizaje ubicuo y móvil, enriquecer documentos escritos con información complementaria en clip de vídeos o podcast de audio, activar entornos lúdicos y motivantes para la formación, o facilitar la observación de los objetos desde múltiples perspectivas y llevando a cabo su selección el estudiante (Barba, Yasaca y Manosalvas, 2015; Fonseca, Redondo y Valls, 2015; Han, Jo, Hyun y So, 2015; Prendes, 2015; Reinoso, 2016; Santos et al, 2016).

Por todo ello, se establece un amplio abanico de posibilidades en el ámbito educativo a través de la incorporación de la RA, y que Bower et al. (2014) ya recogían por ejemplo a través de diferentes herramientas disponibles en la red, y que el docente podía hacer uso e incorporar en su proceso de enseñanza y aprendizaje, algunas de las cuales, y a modo de síntesis, se presentan a través de la Tabla $\mathrm{n}^{\circ} 1$.

Evaluación por y desde los usuarios: objetos de aprendizaje con Realidad Aumentada. Julio Cabero Almenara, Carmen Llorente Cejudo, Juan Jesús Gutiérrez Castillo. 
Tabla no 1.

Herramientas de RA y posibilidades educativas. Adaptado de Bower et al. (2014).

\begin{tabular}{|c|c|c|}
\hline $\begin{array}{l}\text { Centro de Recursos } \\
\text { LearnAR }\end{array}$ & $\begin{array}{l}\text { Ofrece un paquete de } 10 \text { experiencias de } \\
\text { aprendizaje de Realidad Aumentada } \\
\text { basadas en marcadores para la biología, } \\
\text { física, idiomas, inglés, matemáticas y } \\
\text { educación religiosa. }\end{array}$ & http://learnar.org \\
\hline Fetch! Lunch rush! & $\begin{array}{l}\text { Herramientas para ayudar a los } \\
\text { estudiantes a desarrollar habilidades } \\
\text { matemáticas elementales haciéndoles } \\
\text { preguntas y requiriéndoles encontrar un } \\
\text { marcador de Realidad Aumentada con la } \\
\text { respuesta correcta. }\end{array}$ & $\begin{array}{l}\text { http://pbskids.org/apps/fetch- } \\
\text { lunch-rush.html }\end{array}$ \\
\hline Zooburst & $\begin{array}{l}\text { A través de la herramienta los estudiantes } \\
\text { pueden desarrollar historias digitales 3D } \\
\text { mediantes la carga de fotos, texto y/o } \\
\text { audio. }\end{array}$ & http://zooburst.com \\
\hline $\begin{array}{l}\text { MoonGlobe } \\
\text { MarsGlobe }\end{array}$ & $\begin{array}{lcccr}\text { Permiten a los } & \text { estudiantes explorar } \\
\text { aspectos de la luna y Marte para } \\
\text { encontrar los } \\
\text { "habitables". }\end{array}$ & http://www.midnightmartian.com \\
\hline Planets3D & Para planificar aventuras a otros planetas. & https://popartoys.com \\
\hline
\end{tabular}

Así pues, no cabe duda que el soporte que ofrece la utilización de la RA en los contextos educativos es altamente positivo, lo que permite mejorar las acciones formativas para que los alumnos aumenten los niveles de aprendizaje mediante la creación de escenarios tecnológicos. En ese sentido, y así lo apuntan Cabero y Barroso (2016), uno de los aspectos a destacar es que facilita la comprensión de fenómenos y conceptos complejos, ya que favorece, por una parte, la descomposición de un fenómeno y/o objeto en sus diferentes fases, etapas o partes; y por otra, que permite la percepción del objeto o fenómeno desde diferentes puntos de vista.

Quizás una de las grandes limitaciones que presenta la incorporación en las aulas está más ligada al aspecto técnico de la tecnología que al didáctico-curricular. Es el caso de la gran cantidad de fallos que, en algunas ocasiones, ofrece el dispositivo (Tablet, en la mayoría de los casos), o la conexión a internet, así como la dificultad que a ciertas edades presenta el manejo de dispositivos móviles en ambientes típicos de clase. Otros autores, ya apuntaban a una cierta sobrecarga cognitiva del alumnado debido a las altas expectativas multitareas que presentan las actividades basadas en RA (Dunleavy et al., 2009). Y por último, y aunque en la actualidad ya existen empresas que están haciendo más accesible el diseño de objetos de RA, si es cierto que se hace necesario un software altamente especializado.

Pero, lo que realmente revoluciona el mundo de la RA, y por ende el de la educación, es la aparición de diferentes sistemas que permiten a los docentes no solo consultar e incorporar, sino planificar y diseñar objetos de RA que den respuesta a las necesidades particulares que se les plantean en su práctica diaria (Cabero \& García, 2016). Algunos de los ejemplos son: Aurasma (http://aurasma.com), Layar (http://layar.com), y Junaio (http://junaio.com). Por lo tanto, comienza la verdadera transformación en el campo de la RA para profesores y alumnos: pasamos de ser meros consumidores de objetos de RA

Evaluación por y desde los usuarios: objetos de aprendizaje con Realidad Aumentada. Julio Cabero Almenara, Carmen Llorente Cejudo, Juan Jesús Gutiérrez Castillo. 
a planificadores, diseñadores, constructores y administradores de nuestras propias experiencias de RA.

Por último no podemos olvidar que la RA presenta una serie de inconvenientes y dificultades a la hora de su incorporación a la enseñanza, como son: las pocas investigaciones realizadas, la novedad y rapidez de como se está desarrollando la tecnología, los pocos objetos de aprendizaje que se disponen, la dificultad cognitiva el interaccionar en un contexto que mezcla lo real y lo virtual, y la falta de fundamentación teórica para su incorporación.

\subsection{La producción de los Objetos de aprendizaje en Realidad Aumentada.}

Como se ha apuntado con anterioridad, los objetos en RA requieren de la utilización de una diversidad de programas (Layar, Junaio, Augment, Aurasma, Unity,...) (Cabero \& García, 2016), para la realización de diferentes acciones que van desde la producción de objetos específicos como clip de vídeo o audio o materiales específicos en 3D, hasta software específicos para la programación de la interacción de la realidad digital con la realidad física y la combinación de los diferentes objetos.

Los dos objetos que se han producido pertenecían a las disciplinas de "Tecnología Educativa" y "Tecnologías de la Información y Comunicación ampliadas a la Educación", y abordaban en concreto las temáticas de las "formas de utilizar el vídeo en la enseñanza" (vídeo) y el "diseño, producción y evaluación de tecnologías de la información y comunicación a aplicadas a la educación” (“diseño"). Los objetos producidos, los elementos que lo conformaban y los contenidos que se desarrollan, pueden observarse en las siguientes direcciones web: http://intra.sav.us.es/proyectorafodiun/images/pdf/objetos-ra/Roles_video-lanzador.pdf ("vídeo") y http://intra.sav.us.es/proyectorafodiun/images/pdf/objetos-ra/Disenolanzador.pdf ("diseño").

En el objeto del diseño (Figura 1), se analizaban las fases fundamentales del diseño y la producción de cualquier tecnología, y las diferentes estrategias y técnicas que se pudieran utilizar para la evaluación de las TIC, y se incorporaban en él una diversidad de recursos: clip de vídeos, grabaciones en formato polimedia, vídeos transparentes, documentos en pdf, recursos web, y animaciones en 3D. Por el contrario en el de la temática del vídeo (Figura 2) solamente se incluía dos polimedias y se construyó bajo la modalidad de "apuntes enriquecidos con clip del vídeo" (Cabero \& García, 2016). Dicho en otros términos, en el primero se incorporaron más recurso, y era más complejo de manejar por el estudiante, por la diversidad de contenidos que se desarrollaban.

Evaluación por y desde los usuarios: objetos de aprendizaje con Realidad Aumentada. Julio Cabero Almenara, Carmen Llorente Cejudo, Juan Jesús Gutiérrez Castillo. 


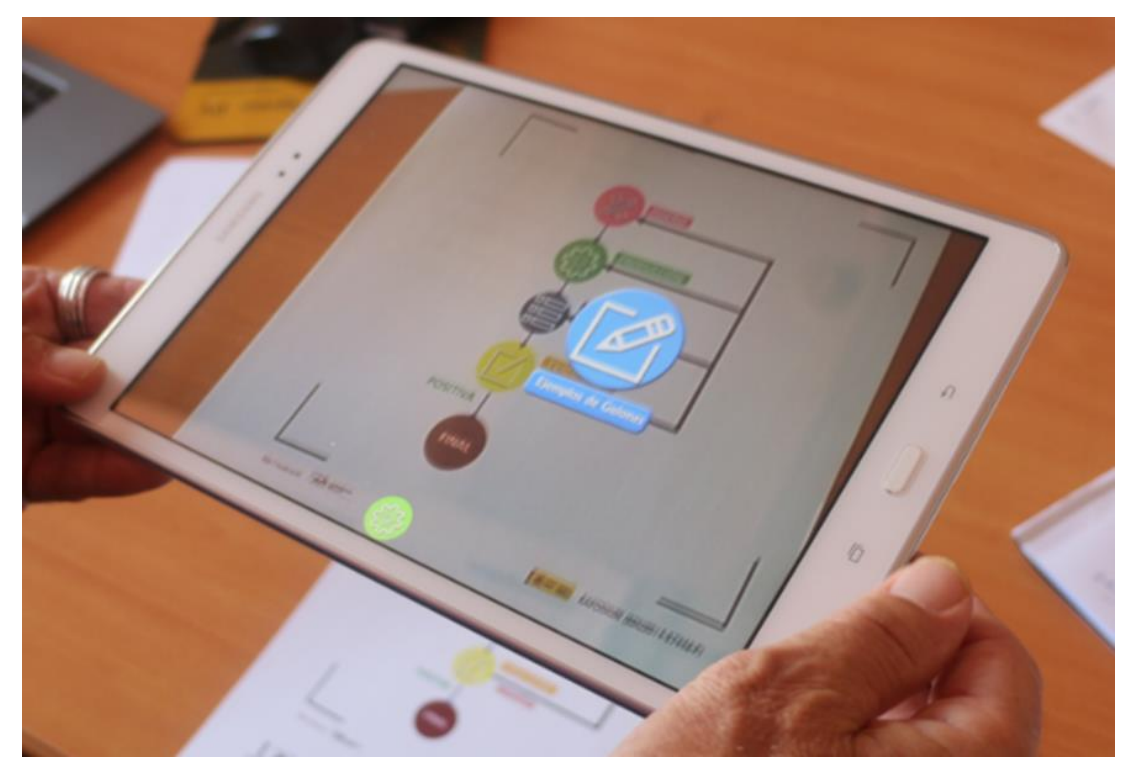

Figura 1. Objetos producidos en RA para el tema del diseño, producción y evaluación de TIC.

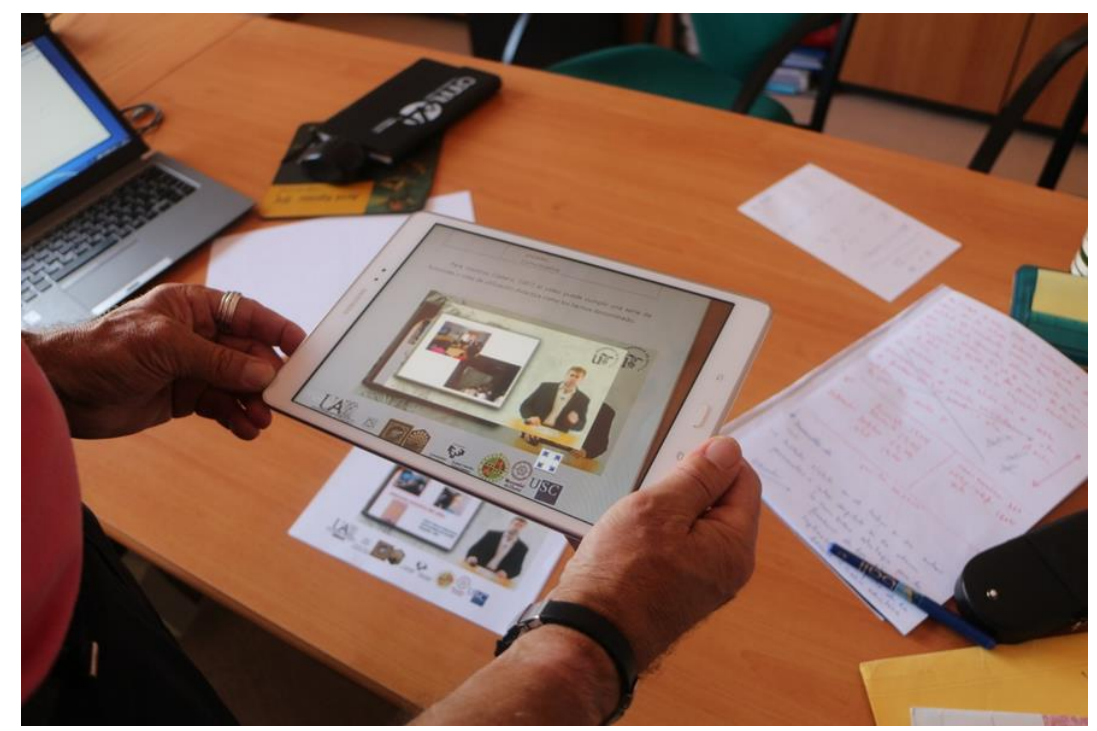

Figura 2. Objetos producidos en RA para el tema de los usos del vídeo.

Podríamos preguntarnos: ¿qué ofrece estos materiales a los estudiantes que cursaban estos estudios? Y las respuestas que podemos ofrecer van en diferentes direcciones: por una se le han enriquecido a los estudiantes los apuntes con diferentes recursos externos que iban desde clip de vídeos específicamente grabados para ello; el acceder en cualquier lugar a la información digital que enriquecía la presentada en la analógica; y la creación de un entorno multimedia y el acceso al mismo con un único dispositivo.

En la Anexo 1, se presenta la relación de programas y software informáticos que se utilizaron para la elaboración de ambos objetos, y la función a los que se destinó cada uno de ellos. Señalar, que los objetos se diseñaron con app para que pudieran ser utilizados tanto en dispositivos IOs como en Android.

Evaluación por y desde los usuarios: objetos de aprendizaje con Realidad Aumentada. Julio Cabero Almenara, Carmen Llorente Cejudo, Juan Jesús Gutiérrez Castillo. 


\section{El estudio realizado.}

Dentro de las diferentes estrategias a utilizar para la evaluación de las tecnologías de la información y comunicación, nos encontramos con la denominada "evaluación por y desde los usuarios" (Barroso, Llorente, \& Cabero, 2015) que es la efectuada directamente por los usuarios destinatarios de las mismas. Y es bajo esta perspectiva desde la que hemos organizado el presente trabajo.

Indicar que uno de los objetivos que perseguía en el proyecto en el cual se inserta el estudio (RAFODIUN - EDU2014-57446-P) era "Diseñar y producir distintos contenidos en formato RA para ser aplicados en contextos de formación universitaria en distintas áreas curriculares, y evaluar sus posibilidades de cara al rendimiento de los alumnos". Pues bien dentro de este objetivo se quiso conocer las valoraciones que los estudiantes que habían utilizado dichos objetos de aprendizaje realizaban de los mismos.

La investigación se llevó a cabo con estudiantes que cursaban las asignaturas de "Tecnología Educativa" y "TIC aplicadas a la educación" cursados en los estudios de Ciencia de la Educación, y Educación Infantil y Primaria de la Facultad de Ciencias de la Educación de la Universidad de Sevilla.

El número total de participantes fue de 429, 145 evaluaron el objeto de aprendizaje denominado "vídeo" y 284 el de "diseño". El estudio realizado podemos considerarlo del tipo "expost-facto" (Mateo, 2004), efectuándose un muestreo no probabilístico y del tipo de conveniencia o causal (Alaminos, 2006; Sabariego, 2012) que se basa en la facilidad de acceso por parte del investigador a los individuos que participaron en el estudio, en este caso los investigadores eran profesores de dichas asignaturas.

El instrumento de recogida de información que se utilizó fue un cuestionario "ad hoc" con construcción tipo Likert, conformado por 13 ítems con seis opciones de respuestas (MP=Muy positivo/Muy de acuerdo (1); $\mathrm{P}=\mathrm{Positivo/De}$ acuerdo (2); R+=Regular positivo/Moderadamente de acuerdo (3); $\mathrm{R}-=$ Regular negativo/Moderadamente en desacuerdo (4); N=Negativo /En desacuerdo (5); y MN=Muy negativo/muy en desacuerdo (6)), que perseguían recoger información sobre tres dimensiones: aspectos técnicos y estéticos del objeto producido en RA (4 ítems), facilidad de utilización (7 ítems) y guía elaborada para facilitar la comprensión del funcionamiento del objeto por los estudiantes. El instrumento se construyó a partir de otros realizados para otras investigaciones donde los estudiantes evaluaron diferentes materiales educativos como eran multimedias o para la formación virtual (Cabero \& Llorente, 2009; Llorente \& Cabero, 2013; Cabero \& Marín, 2013; Marín, Cabero \& Barroso, 2014), y fue administrado vía Internet.

La guía ofrecía a los estudiantes información respecto a diferentes aspectos: lugar desde el cual podrían bajarse la app para la utilización del programa, dispositivos que podrían utilizar para la interacción con el objeto, y recomendaciones para la observación de vídeos demostrativos del funcionamiento que se realizaron para facilitar la comprensión de su utilización y manejo. En la Figura 3, se ofrece una imagen de la guía elaborada para el objeto de "Vídeo".

Evaluación por y desde los usuarios: objetos de aprendizaje con Realidad Aumentada. Julio Cabero Almenara, Carmen Llorente Cejudo, Juan Jesús Gutiérrez Castillo. 


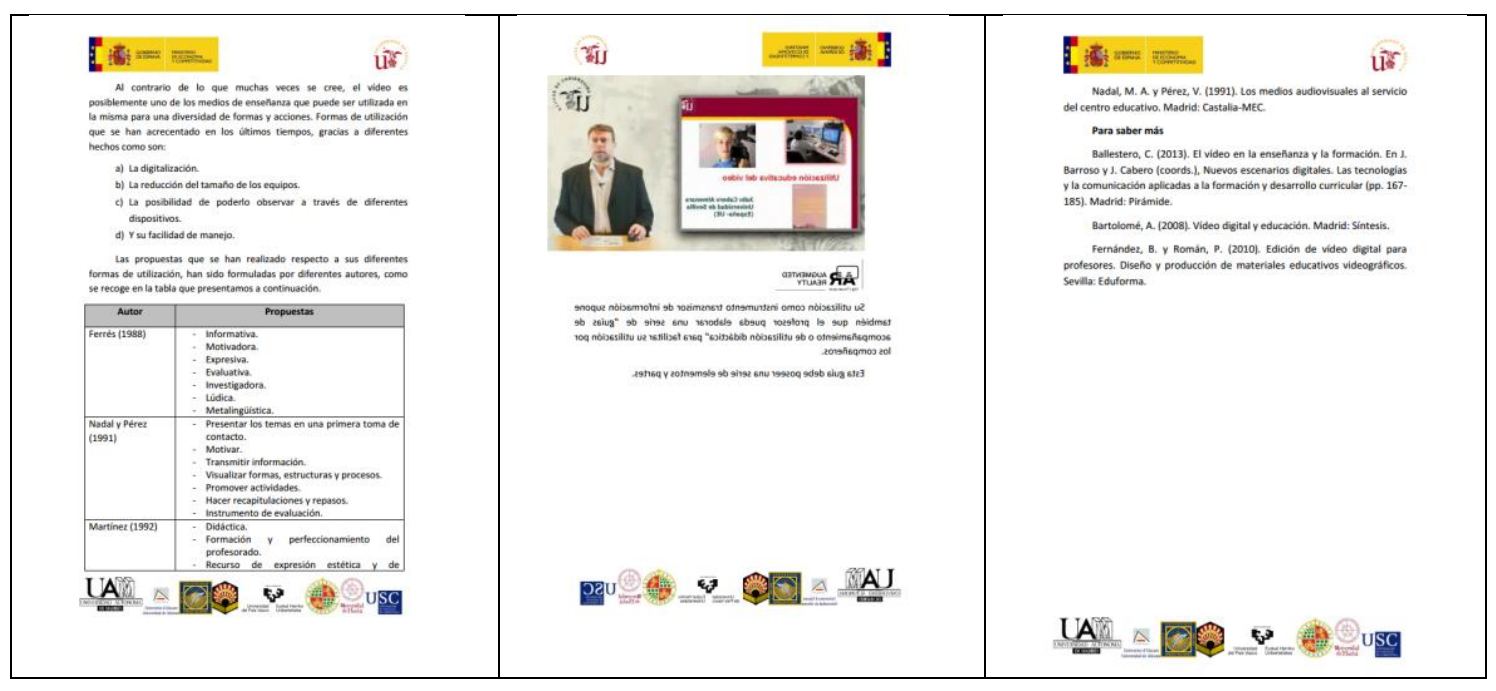

Figura 3. Guía del objeto "Vídeo".

La obtención del índice de fiabilidad del instrumento se obtuvo mediante la alfa de Cronbach como sugieren O’Dwyer y Bernauer (2014) para este tipo de instrumentos, alcanzándose los valores, para la globalidad del instrumento y para cada una de las dimensiones que lo conforman, que se presentan en la Tabla 2.

Tabla 2.

Alfa de Cronbach para la Evaluación de la calidad del objeto estudiado.

\begin{tabular}{|l|l|}
\hline \multicolumn{2}{|l|}{ Evaluación de la calidad del objeto } \\
\hline Dimensión & Valor alfa \\
\hline Total instrumento & 0,951 \\
\hline Aspectos técnicos & 0,893 \\
\hline Facilidad de navegación & 0,900 \\
\hline Tutorial del programa & 0,882 \\
\hline
\end{tabular}

Valores que de acuerdo con diferentes autores (Mateo, 2004; O’Dwyer \& Bernauer, 2014) pueden considerarse de altos o muy altos, y denotarían un nivel aceptado de fiabilidad del instrumento.

Una vez que se le había explicado en el aula a los estudiantes en qué consistía la RA, el procedimiento que se siguió para la evaluación de los materiales por ellos consistió en una serie de etapas: demostración del funcionamiento de los objetos, presentación del lugar del cual podrían bajarse las distintas guías, trabajo individual de los alumnos con sus dispositivos móviles durante dos semanas con los objetos, y administración del cuestionario. Señalar que la situación en la cual se incorporaron los objetos de RA, fueron de situaciones reales de enseñanza, pues los alumnos serían evaluados de los contenidos desarrollando en ambos objetos. 


\section{Resultados encontrados.}

Tras la realización de la experiencia obtuvimos los resultados que se presentan en la Tabla 3, en ella se ofrecen los valores medios y las desviaciones típicas obtenidas en cada uno de los ítems del instrumento, en su globalidad y en las tres dimensiones que lo conforman. Al mismo tiempo los resultados se presentan diferenciados, tanto para los dos objetos de aprendizaje elaborados, como para la suma de los dos.

Tabla 3.

Puntuaciones medias y desviaciones típicas totales alcanzadas para cada una de las dimensiones.

\begin{tabular}{|c|c|c|c|c|c|c|}
\hline & \multicolumn{2}{|c|}{ Vídeo } & \multicolumn{2}{|c|}{ Diseño } & \multicolumn{2}{|c|}{ Conjunta } \\
\hline & $\mathrm{m}$ & d.t & $\mathrm{m}$ & d.t & $\mathrm{m}$ & d.t \\
\hline \multicolumn{7}{|l|}{ 1. Aspectos técnicos y estéticos } \\
\hline $\begin{array}{l}\text { 1.1. El funcionamiento del recurso } \\
\text { en RA que te hemos presentado } \\
\text { es: }\end{array}$ & 2,21 & 1,05 & 2,17 & 95 & 2,18 & ,98 \\
\hline $\begin{array}{l}1.2 \text { En general, la estética del } \\
\text { recurso producido en RA lo } \\
\text { consideras: }\end{array}$ & 2,44 & ,96 & 2,21 & ,97 & 2,29 & ,97 \\
\hline $\begin{array}{l}\text { 1.3 En general, el funcionamiento } \\
\text { técnico del recurso producido en } \\
\text { RA lo calificaría de: }\end{array}$ & 2,46 & 1,04 & 2,37 & 1,01 & 2,40 & $\begin{array}{l}1,0 \\
1\end{array}$ \\
\hline $\begin{array}{l}\text { 1.4. En general, cómo valorarías } \\
\text { la presentación de la información } \\
\text { en la pantalla: }\end{array}$ & 2,28 & 1,02 & 2,32 & ,99 & 2,31 & $\begin{array}{l}1,0 \\
0\end{array}$ \\
\hline \multicolumn{7}{|l|}{ 2. Facilidad de utilización } \\
\hline $\begin{array}{l}\text { 2.1. Cómo calificará la facilidad } \\
\text { de utilización y manejo del } \\
\text { recurso en RA presentado: }\end{array}$ & 2,40 & 1,02 & 2,33 & 1,01 & 2,35 & $\begin{array}{l}1,0 \\
1\end{array}$ \\
\hline $\begin{array}{l}\text { 2.2. Cómo calificarías la facilidad } \\
\text { de comprensión } \\
\text { funcionamiento técnico del } \\
\text { recurso en RA presentado: }\end{array}$ & 2,43 & 1,00 & 2,40 & 1,07 & 2,41 & $\begin{array}{l}1,0 \\
4\end{array}$ \\
\hline $\begin{array}{l}\text { 2.3. Desde tu punto de vista, } \\
\text { cómo valoraría el diseño general } \\
\text { del recurso en RA elaborado: }\end{array}$ & 2,38 & ,98 & 2,30 & 1,03 & 2,32 & $\begin{array}{l}1,0 \\
1\end{array}$ \\
\hline $\begin{array}{lcr}\text { 2.4. Desde tu punto de } & \text { vista, } \\
\text { cómo } & \text { valoraría } & \text { la } \\
\text { accesibilidad/usabilidad } & \text { del } \\
\text { recurso en RA presentado: } & \\
\end{array}$ & 2,52 & 1,10 & 2,51 & 1,10 & 2,51 & $\begin{array}{l}1,1 \\
0\end{array}$ \\
\hline $\begin{array}{l}\text { 2.5. Desde tu punto de vista, } \\
\text { cómo valoraría la flexibilidad de } \\
\text { utilización del material en RA } \\
\text { presentado: }\end{array}$ & 2,57 & 1,04 & 2,51 & 1,08 & 2,53 & $\begin{array}{l}1,0 \\
6\end{array}$ \\
\hline $\begin{array}{l}\text { 2.7. El utilizar el recurso en RA } \\
\text { producido te fue divertido: }\end{array}$ & 2,34 & 1,22 & 2,35 & 1,13 & 2,34 & $\begin{array}{l}1,1 \\
6\end{array}$ \\
\hline
\end{tabular}

Evaluación por y desde los usuarios: objetos de aprendizaje con Realidad Aumentada. Julio Cabero Almenara, Carmen Llorente Cejudo, Juan Jesús Gutiérrez Castillo. 


\begin{tabular}{|c|c|c|c|c|c|c|}
\hline \multicolumn{7}{|l|}{ 3. Guía / tutorial del programa } \\
\hline $\begin{array}{l}\text { 3.1. En general, cómo calificaría } \\
\text { de eficaz y comprensible la } \\
\text { información ofrecida para } \\
\text { manejar el recurso en RA } \\
\text { presentado: }\end{array}$ & 2,31 & ,92 & 2,21 & 97 & 2,25 & 95 \\
\hline $\begin{array}{l}\text { 3.2. La información ofrecida para } \\
\text { manejar el recurso en RA te fue } \\
\text { simple y comprensible. }\end{array}$ & 2,32 & 1,01 & 2,30 & 1,07 & 2,31 & $\begin{array}{l}1,0 \\
5\end{array}$ \\
\hline ASPECTOS TÉCNICOS & 2,35 & ,85 & 2,27 & ,83 & 2,29 & ,84 \\
\hline $\begin{array}{l}\text { FACILIDAD } \\
\text { NAVEGACIÓN }\end{array}$ & 2,44 & ,85 & 2,40 & ,86 & 2,41 & ,85 \\
\hline $\begin{array}{l}\text { TUTORIAL } \\
\text { PROGRAMA }\end{array}$ & 2,31 & 91 & 2,26 & ,95 & 2,28 & ,93 \\
\hline TOTAL INSTRUMENTO & 2,39 & ,80 & 2,33 & ,80 & 2,35 &, 80 \\
\hline
\end{tabular}

Como se observa en la Tabla 3, tanto en el total del instrumento, como en las diferentes dimensiones, como en sus ítems, las puntuaciones medias alcanzadas se sitúan en el intervalo 2,18-2,57, lo que supone una valoración de los objetos como positivos, tanto en sus "aspectos técnicos" $(2,29)$, "facilidad de navegación" $(2,41)$ y el "tutorial del programa". En el sumatorio de la valoración de los objetos, la puntuación más significativa se encontró en el ítem: "El funcionamiento del recurso en RA que te hemos presentado es:" $(2,18)$ y la más baja en: "2.5. Desde tu punto de vista, cómo valoraría la flexibilidad de utilización del material en RA presentado:" (2,53); es decir, en ningún caso se alcanzó el nivel medio central de "3".

Por otra parte, si observamos las puntuaciones de la tabla $\mathrm{n}^{\mathrm{o}} 2$ nos daremos cuenta que no hay dispersión en las valoraciones medias alcanzadas en los diferentes ítems, tanto de manera global como en cada uno de los objetos.

Con el objeto de conocer si había diferencias significativas en las valoraciones que los estudiantes realizaron de ambos objetos, formulamos las siguientes hipótesis:

- Hipótesis nula (H0): No existen diferencias significativas en las valoraciones que los alumnos realizan de ambos objetos de RA, con un riesgo alfa de equivocarnos del 0.05 , tanto en lo que se refiere a las valoraciones asignada a la globalidad del objeto, como en sus aspectos técnicos, facilidad de navegación y tutorial del programa.

- Hipótesis alternativas (H1): No existen diferencias significativas en las valoraciones que los alumnos realizan de ambos objetos de RA, con un riesgo alfa de equivocarnos del 0.05 , tanto en lo que se refiere a las valoraciones asignada a la globalidad del objeto, como en sus aspectos técnicos, facilidad de navegación y tutorial del programa.

Para ello aplicamos el estadístico t de Student para muestras independientes, realizando previamente la prueba de Levene para el contraste de la igualdad de las varianzas, para

Evaluación por y desde los usuarios: objetos de aprendizaje con Realidad Aumentada. Julio Cabero Almenara, Carmen Llorente Cejudo, Juan Jesús Gutiérrez Castillo. 
que en función de dicho valor determinar el valor t que deberíamos adoptar. En la Tabla 4, se presentan los valores " $t$ " obtenidos y el nivel de significación que nos permitía aceptar o rechazar la $\mathrm{H} 0$ formulada.

Tabla 4.

Valores de prueba Levene y t de Student.

\begin{tabular}{|c|c|c|c|c|c|c|}
\hline & \multicolumn{5}{|c|}{ Prueba de Levene } \\
\hline & & \multirow{3}{*}{$\frac{F}{1,075}$} & \multirow{3}{*}{$\frac{\text { Sig. }}{, 784}$} & \multirow{2}{*}{$\frac{T}{915}$} & \multirow{2}{*}{$\frac{\mathrm{Gl}}{427}$} & Sig. \\
\hline $\begin{array}{l}\text { Aspectos } \\
\text { técnicos }\end{array}$ & $\begin{array}{l}\text { Se asumen varianzas } \\
\text { iguales }\end{array}$ & & & & &, 361 \\
\hline & $\begin{array}{l}\text { No se asumen varianzas } \\
\text { iguales }\end{array}$ & & & 905 & 281,95 &, 366 \\
\hline $\begin{array}{l}\text { Facilidad de } \\
\text { navegación }\end{array}$ & $\begin{array}{ll}\text { Se asumen varianzas } \\
\text { iguales }\end{array}$ &, 034 & 855 & & 427 &, 631 \\
\hline & $\begin{array}{l}\text { No se asumen varianzas } \\
\text { iguales }\end{array}$ & & &, 482 & 293,52 &, 630 \\
\hline $\begin{array}{l}\text { Tutorial del } \\
\text { programa }\end{array}$ & $\begin{array}{ll}\text { Se asumen varianzas } \\
\text { iguales }\end{array}$ & 1,260 & 262 &, 595 & 427 &, 552 \\
\hline & $\begin{array}{l}\text { No se asumen varianzas } \\
\text { iguales }\end{array}$ & & &, 603 & 301,05 &, 547 \\
\hline $\begin{array}{l}\text { Total del } \\
\text { instrument }\end{array}$ & $\begin{array}{ll}\text { Se asumen varianzas } \\
\text { iguales }\end{array}$ &, 052 & 820 &, 690 & &, 491 \\
\hline o & $\begin{array}{l}\text { No se asumen varianzas } \\
\text { iguales }\end{array}$ & & &, 692 & 292,93 &, 489 \\
\hline
\end{tabular}

Como se puede observar los valores $\mathrm{t}$ de Student alcanzados no permiten rechazar ninguna de las H0 formuladas; en consecuencia podemos decir que no existen diferencias significativas en las valoraciones que los alumnos realizan respecto a ambos objetos. Dicho en otros términos ambos objetos recibieron las mismas valoraciones positivas.

Indicar, aunque no se profundizará para no extenderse en el tema, que idénticos resultados se encontraron cuando se aplicó el estadístico de Student a los diferentes ítems que conformaban el instrumento.

\section{Conclusiones, discusión y limitaciones del estudio.}

Una de las primeras conclusiones del trabajo es que la utilización de objetos de RA en la formación universitaria, despierta verdadero interés por los estudiantes, y en consonancia nuestros resultados apoyan los elaborados por diferentes autores cuando nos hablan que los alumnos muestran altos niveles de satisfacción cuando utilizan esta tecnología y los niveles de motivación que manifiestan cuando están inmersos en acciones formativas de este tipo son significativos (Chin-Ming \& Yen Nung, 2011 De la Torre, Martín-Dorta, Saorín, Carbonel, \& Contero, 2013; Kamarainen et al., 2013; Ko,

Evaluación por y desde los usuarios: objetos de aprendizaje con Realidad Aumentada. Julio Cabero Almenara, Carmen Llorente Cejudo, Juan Jesús Gutiérrez Castillo. 
Chang, Chen, \& Hua, 2011; Neven, Hala, \& Mohamed, 2011; Di Serio, Ibáñez, \& Delgado 2013; Wojciechowski \& Cellary, 2013; Cózar, De Moya, Hernández, \& Hernández, 2015; Santos et al., 2016; Ericson et al., 2016; Kim, Hwang, \& Zo, 2016).

Nuestros resultados avalan la forma en la cual se produjeron los objetos de aprendizaje en RA, y ello tanto desde los puntos de vista técnico, estético y de facilidad de utilización. Por otra parte, creemos que la construcción de una guía que facilite la comprensión al estudiante, tanto de la información que se encontrará, como de la app que debe instalarse y la manera en la que se aconseja su utilización, ha facilitado el proceso de utilización del recurso de aprendizaje. Como señalan Garay, Tejada y Castaño (2017: 158), la actitud hacia el objeto:

"viene mediada por la percepción en relación a la facilidad de uso del objeto educativo. De ahí la importancia del diseño tanto desde el punto de vista de la educación a las necesidades del alumnado como a que sea usable y atractivo."

Las valoraciones positivas realizadas por parte de los estudiantes, nos permiten señalar que estos recursos pueden ser válidos para su incorporación en los procesos de enseñanza-aprendizaje, y ello viene avalado por diferentes aspectos, como son: el hecho de no requerir tecnología adicional de incorporación ya que los estudiantes a este nivel cuentan con dispositivos móviles (smartphone y tablet) que facilitan su utilización y no requieren incorporar al aula tecnología adicional, y que la competencia digital de los estudiantes para el manejo de estos instrumentos tecnológicos es elevada y por tanto no les presentan dificultad para su utilización.

Por otra parte, el hecho de que resultados similares se encontraron con diferentes tipos de objetos y en grupos de alumnos diferentes, y pertenecientes a estudios distintos; hacen que los hallazgos puedan ser más generalizables. Por otra parte, no se debe olvidar que los hallazgos encontrados han sido obtenidos en situaciones reales de docencia, pues los contenidos presentados pertenecían a los programas oficiales de las asignaturas y de ellos los alumnos serían evaluados.

El estudio refuerza los planteamientos de Wojciechowski y Cellary (2013), que manifiestan que con estos recursos de aprendizaje los alumnos se muestran competentes para interactuar con los objetos virtuales en una forma directa y natural a través de la manipulación de objetos reales, no requiriendo dispositivos adicionales, que aunque están disminuyendo su costo, siguen siendo elevados, y algunas veces su manejo son complejos.

Las valoraciones positivas de estos objetos por parte de los estudiantes que hemos encontrado en nuestro estudio, coinciden con las encontradas por otros autores (Barroso \& Cabero, 2016; Garay, Tejada, \& Maiz, 2017; Garay, Tejada, \& Castaño, 2017), que han utilizado también objetos de RA en contextos de formación universitaria.

También nos gustaría señalar que los niveles de fiabilidad alcanzados con el instrumento tanto de manera general, como en sus diferentes dimensiones, indican que es de utilidad para recoger información de los alumnos respecto a la evaluación que

Evaluación por y desde los usuarios: objetos de aprendizaje con Realidad Aumentada. Julio Cabero Almenara, Carmen Llorente Cejudo, Juan Jesús Gutiérrez Castillo. 
realizan de objetos de aprendizaje con construcción en formato RA. Al mismo tiempo su simplicidad y rapidez hace que pueda ser fácilmente administrado para recoger información que permita que los estudiantes evalúen recursos producidos en formato RA.

Respecto a líneas futuras de investigación que se pueden abrir, son diversas: la producción de objetos de aprendizaje en RA a otras áreas de conocimiento diferentes a la utilizada en este estudio, producir objetos que incorporen recursos distintos a los ubicados en los objetos aquí producidos, y analizar y relacionar la evaluación efectuada por los estudiantes con otras variables como pueden ser niveles de satisfacción por participar en experiencias de aprendizaje de este tipo o el rendimiento de aprendizaje. De todas formas debemos reconocer que el trabajo abre una línea de investigación sobre estos materiales didáctico, donde por ahora ha existido más un planteamiento $\mathrm{y}$ reflexión tecnológica, que pedagógica y educativa.

Presentación del artículo: 13 de febrero de 2017

Fecha de aprobación: 15 de marzo de 2017

Fecha de publicación: 31 de marzo de 2017

Cabero Almenara, J., Llorente Cejudo, M.C., \& Gutiérrez-Castillo, J.J. (2017). Los alumnos como evaluadores de objetos de aprendizaje en Realidad Aumentada. RED. Revista de Educación a Distancia, 53. Consultado el (dd/mm/aaaa) en http://www.um.es/ead/red/53/cabero_et_al.pdf

\section{FINANCIACIÓN}

El presente trabajo se inserta en el proyecto de investigación denominado "Realidad Aumentada para Aumentar la Formación. Diseño, Producción y Evaluación de Programas de Realidad Aumentada para la Formación Universitaria (RAFODIUN) (EDU2014-57446-P).

\section{Referencias bibliográficas.}

Alaminos, A. (2006). El muestreo en la investigación social. En A. Alaminos y J.L. Castejón. Elaboración, análisis e interpretación de encuestas, cuestionarios y escalas de opinión (pp. 46-67). Alcoy: Marfil.

Bacca, J., Baldiris, S., Fabregat, R., Graf, S., \& Kinshuk, J. (2014). Augmented Reality Trends in Education: A Systematic Review of Research and Applications. Educational Technology \& Society, 17(4), 133-149.

Barba, R., Yasaca, S., \& Manosalvas, C. (2015). Impacto de la realidad aumentada móvil en el proceso enseñanza-aprendizaje de estudiantes universitarios del área de medicina. En AIDIPE (ed.). Investigar con y para la Sociedad (pp. 14211429), Cádiz: Bubok Publishing S.L.

Evaluación por y desde los usuarios: objetos de aprendizaje con Realidad Aumentada. Julio Cabero Almenara, Carmen Llorente Cejudo, Juan Jesús Gutiérrez Castillo. 
Barroso, J. \& Cabero, J. (2016). Evaluación de objetos de aprendizaje en realidad aumentada: estudio piloto en el grado de Medicina. Enseñanza \& Teaching, 34(2), 149-167.

Barroso, J., Llorente, M.C., \& Cabero, J. (2015). El diseño, y la producción y la evaluación de TIC aplicadas a los procesos de Enseñanza-aprendizaje. En Cabero, J. y Barroso, J. (cords.). Nuevos retos en tecnología educativa (pp.69-85). Madrid: Síntesis.

Billinghurst, M., \& Duenser, A. (2012). Augmented reality in the classroom. Computer, $45,56-63$.

Bower, M. et al. (2014). Augmented Reality in education - cases, places and potentials. Educational Media International, 51(1), 1-15.

Cabero Almenara, J. \& Barroso Osuna, J. (2016). Posibilidades educativas de la realidad aumentada. New Approaches in Educational Research, 5(1), 46-52. doi: 10.7821/naer.2016.1.140

Cabero, J. \& García, F. (coords.) (2016). Realidad aumentada. Tecnología para la formación. Madrid: Síntesis.

Cabero, J. \& Llorente, M.C. (2009). Actitudes, satisfacción, rendimiento académico y comunicación online en procesos de formación universitaria en "blended learning". Revista Electrónica Teoría de la Educación: Educación y Cultura en la Sociedad de la Información, 10(1), 172-189.

Cabero, J. \& Marín, V. (2013). Valoración del entorno formativo universitario Dipro 2.0. Profesorado. Revista de Currículum y formación del profesorado, 17(2), 369383.

Chin-Ming, C. \& Yen Nung, T. (2011). Interactive augmented reality system for enhancing library instruction in elementary schools. Computers and education, 59, 638-652.

Cózar, R., De Moya, M., Hernández, J., \& Hernández, J. (2015). Tecnologías emergentes para la enseñanza de las Ciencias Sociales. Una experiencia con el uso de Realidad Aumentada en la formación inicial de maestros. Digital Education Review, 27, 138-153.

De la Torre Cantero, J., Martín-Dorta, N., Saorín Pérez, J. L., Carbonel Carrera, C., \& Contero González, M. (2013). Entorno de aprendizaje ubicuo con realidad aumentada y tabletas para estimular la comprensión del espacio tridimensional. RED, Revista de Educación a Distancia, 37, 1-17. Recuperado de: http://www.um.es/ead/red/37.

Evaluación por y desde los usuarios: objetos de aprendizaje con Realidad Aumentada. Julio Cabero Almenara, Carmen Llorente Cejudo, Juan Jesús Gutiérrez Castillo. 
Dede, C. (2009). Immersive interfaces for engagement and learning. Science, 323, 6669.

Di Serio, A., Ibáñez, M. B., \& Delgado, C. (2013). Impact of an augmented reality system on students' motivation for a visual art course. Computers \& Education, 68, 586-596. doi:10.1016/j.compedu.2012.03.002

Dunleavy, M., Dede, C., \& Mitchell, R. (2009). Affordances and limitations of immersive participatory augmented reality simulations for teaching and learning. Journal of Science Education and Technology, 18(1), 7-22. doi:10.1007/s10956008-9119-1.

Ericson, M. et al. (2016). Augmented reality as multimedia: the case for situated vocabulary learning. Research and Practice in Technology Enhanced Learning, 11(4), 1-23. doi: 10.1186/s41039-016-0028-2.

Fonseca, D., Redondo, E., \& Valls, F. (2016). Motivación y mejora académica utilizando realidad aumentada para el estudio de modelos tridimensionales arquitectónicos. Education in the Knowledge Society, EKS, 17(1) 4564. http://dx.doi.org/10.14201/eks20161714564

Garay, U., Tejada, E., \& Castaño, C. (2017). Percepciones del alumnado hacia el aprendizaje mediante objetos educativos enriquecidos con realidad aumentada. Edmetic. Revista de Educación Mediática y TIC, 6(1), 145-164.

Garay, U., Tejada, E., \& Maiz, I. (2017). Valoración de objetos educativos enriquecidos con realidad aumentada: una experiencia con alumnado de máster universitario. Pixel-Bit. Revista de Medios y Educación, 50, 19-31.

Han, J. Jo, M., Hyun, E. \& So, H. (2015). Examining young children's perception toward augmented reality-infused dramatic play. Education Technology Research Development, 63, 455-474. doi:10.1007/s11423-015-9374-9

Johnson, L., Adams, S., \& Cummins, M. (2012). NMC Horizon report: 2012 K-12 edition. Austin, TX: T.N.M. Consortium.

Johnson, L., Smith, R., Levine, A., \& Haywood, K. (2010). The 2010 Horizon report: Australia-New Zealand ed. Austin, TX: T.N.M. Consortium.

Kamarainen, A., Metcalf, Sh., Grotzer, T., Browne, A., Mazzuca, D., Tutwiler, M., \& Dede, Ch. (2013). EcoMOBILE: Integrating augmented reality and probeware with environmental education field trips. Computers \& Education, 68, 545-556. doi:10.1016/j.compedu.2013.02.018

Kaufmann, H., \& Schmalstieg, D. (2003). Mathematics and geometry education with collaborative augmented reality. Computers \& Graphics, 27, 339-345.

Evaluación por y desde los usuarios: objetos de aprendizaje con Realidad Aumentada. Julio Cabero Almenara, Carmen Llorente Cejudo, Juan Jesús Gutiérrez Castillo. 
Kim. K., Hwang, J., \& Zo, H. (2016): Understanding users' continuance intention toward smartphone augmented reality applications. Information Development, $32(2), 161-174$.

Ko, Ch-H., Chang, T., Chen, Y., \& Hua, L. (2011). The Application of Augmented Reality to Design Education. In M. Chang, W.-Y. Hwang, M.-P. Chen, \& W. Müller (Eds.), Edutainment Technologies. Educational Games and VirtualReality/Augmented Reality Applications (pp. 20-24). Heidelberg Berlin: Springer.

Llorente, M.C. \& Cabero, J. (2013). Blended learning: attitudes, satisfaction, academic performance and online communication in processes of University Training. The New Educational Review, 31 (1), 28-39.

Marín, V., Cabero, J., \& Barroso, J. (2014). Evaluando los entornos formativos online. El caso de DIPRO 2.0. REDU. Revista de docencia universitaria, 12 (2), 375-399.

Mateo, J. (2004). La investigación ex-post-facto. In R. Bisquerra (coord.), Metodología de la investigación educativa (pp. 195-230). Madrid: La Muralla.

Neven A. M., Hala, H., \& Mohamed, I. (2011). ARSC: Augmented Reality Student Card An Augmented reality Solution for the educational field. Computers and Education, 56, 1045-1061. doi:10.1016/j.compedu.2010.10.019.

O’Dwyer, L. \& Bernauer, J. (2014). Quantitative research for the qualitative researcher. California: Sage.

Prendes, C. (2015). Realidad aumentada y educación: análisis de experiencias prácticas. Pixel-Bit. Revista de Medios y Educación, 46, 187-203.

Reinoso, R. (2016). Realidad aumentada posibilidades y usos educativos, En Baldiris, S. y otros (eds). Recursos Educativos Aumentados Una oportunidad para la inclusión (pp. 8-29). Colombia: Sello Editorial Tecnolkógico Comfenalco.

Sabariego, M. (2012). El proceso de investigación. En Bisquerra, R. (coord.). Metodología de la investigación educativa. (pp. 127 - 163). Madrid: La Muralla.

Santos, M. E. C., Chen, A., Taketomi, T., Yamamoto, G.,Miyazaki, J., \& Kato, H. (2014). Augmented reality learning experiences: survey of prototype design and evaluation. IEEE Transactions on Learning Technologies, 7(1), 38-56.

Santos, M. et al. (2016). Augmented reality as multimedia: the case for situated vocabulary learning. Research and Practice in Techology Enhanced Learning, 11(4), 1-23. DOI 10.1186/s41039-016-0028-2.

Shelton, B. E. (2002). Augmented reality and education: Current projects and the potential for classroom learning. New Horizons for Learning, 9. Recuperado de http://inst.usu.edu/ bshelton/resources/shelton-NH_aug_real.pdf.

Evaluación por y desde los usuarios: objetos de aprendizaje con Realidad Aumentada. Julio Cabero Almenara, Carmen Llorente Cejudo, Juan Jesús Gutiérrez Castillo. 
Vázquez-Cano E. \& Sevillano, M.L. (edts.) (2015). Dispositivos digitales móviles en educación. El aprendizaje ubicuo. Madrid: Narcea.

Wojciechowski, R. \& Cellary, W. (2013). Evaluation of learners' attitude toward learning in ARIES augmented reality environments. Computers \& Education, 68, 570-585. doi:10.1016/j.compedu.2013.02.014

Evaluación por y desde los usuarios: objetos de aprendizaje con Realidad Aumentada. Julio Cabero Almenara, Carmen Llorente Cejudo, Juan Jesús Gutiérrez Castillo. 